\title{
An Efficient Shearlet Bayesian Network based Approach for Image Denoising
}

\author{
Anubhuti Sharma \\ Samrat Ashok \\ Technological Institute \\ Department of Information \\ Technology, Vidisha, India
}

\author{
Shaila Chugh \\ Samrat Ashok \\ Technological Institute \\ Department of Information \\ Technology, Vidisha, India
}

\begin{abstract}
The evolution innermost the processing power of electronic devices direct the research of well-organized image denoising technique in the direction of the extra complex method which use the difficult transform functional test with statistics. Even supposing with the difficulty of the newly developed techniques, generally algorithm fails to whole adorable stage of performance. For the mainly part algorithms fail since the expedient model mismatch the algorithm presumption taken at the time of improvement. This paper presents a proficient approach intended for image denoising based on Shearlet transform and the Bayesian Network. The projected technique use the geometric dependencies in the shearlet domain in the direction of the Bayesian Network which is next used for predict the noise probability. The Shearlet transform provide improved approximation particularly in different scales, and directional discontinuities which make it preferable designed used within support of processing the pixel around the edge. The later result prove that the future technique better wavelet base method visually and mathematical in conditions of PSNR (peak signal -to -noise ratio).
\end{abstract}

\section{Keywords}

Bayesian Network, Image Denoising, Shearlet Transform

\section{INTRODUCTION}

The denoising of image corrupted by noise is a classical trouble innermost the field of image processing. The requirements of efficient image recovery have continuously rising with the massive production of digital multimedia contents, frequently establish to unwanted noises. Even with first-class quality CCD sensors an image enhancement is for all time essential to extend their quality. The noise could be generated by other sources, such as sensor abnormality or imperfection, thermal noise, and quantization noise for the duration of the digitization process. Generally the denoising technique is classified according to the processing domains it performs, which largely part it within spatial domain and transform domain. A spatial domain is generally choose for low computational complexity resource requirements and quick procedure but it does not present extra ordinary result instead the transform domain method provide improved results at extra processing cost. But the improvement in semiconductor technology and processing architecture create the transform domain method extremely sufficient and so boosted the advance of new transform domain method. The Transform domain techniques highly practicable and therefore boosted the expansion of new transform domain techniques the basic concept behind the transform domain filtering is to transfer the image into suitable domain where the noise components could be recognized or characterized by some inherent aspect of the domain. Lately the wavelet domain technique is gained considerable applicability backed with the big performance. The wavelet transform generate the coefficients at different scale which are then filtered by the different approach. Although the wavelet performs well but it has certain limits like angular property (that means wavelet cannot calculate angles with respect to different orientations) and required superior number of coefficients. The Shearlet is designed to improving these limitations of wavelet. The Shearlet Transform has an angular property and very efficient directionality property, its basis function has many directions, as compare to wavelet which has only three dimensions. It also scales basis functions at different aspect ratios, while wavelet only has the aspect ratio equals to 1 . Because both techniques follow some parallel basic concepts, several processing method designed for wavelet can be adapted for Shearlet without any modifications. The remainder of this paper is arranged as follows. After a brief review of the literature work in section, the III and IV sections give detail essentials of shearlet and Bayesian network respectively. In section $\mathrm{V}$ the proposed algorithm is discussed follow with the simulation results plus conclusion in section VI and VII correspondingly.

\section{RELATED WORK}

This section presents an overview of some recent literature associated to image denoising which found useful during the writing of this paper. [1], this paper is a total different based on multivariate shearlet adaptive shrinkage is future for discontinuity-preserve image denoising. The multivariate adaptive threshold is working to reduce the noise. [2], shearlets are used in image denoising, sparse image representation and edge detection. This paper is used to shearlet transform include the features of directionality, anisotropy and multiscale, it is introduce into image fusion to get complex image. The image could be decomposed by shearlet transform in every scale and every direction, and the aspect information can be fixed minimally. [3], this paper is a unique discrete multistage directional representation called discrete shearlet transform. This progress, which is founded on the shearlet transform, merges the power of multiscale method with a single capability to capture the geometry of multidimensional data and optimally proficient in instead of Threshold is then apply to the images contain edges. Here explain two different method of implementing the shearlet transform. [4], this paper, are proposed in here a novel improvement algorithm used for medical image processing derived from Shearlet transform and unsharp masking. There are four steps in this algorithm. First, histogram equalization is useful to the medical image; second the medical image is decay into low frequency element among high frequency component use shearlet transform. Third, the adaptive thresholding denoising and linear improvement is 
apply to the high frequency components as the low frequency components are not process; Lastly, the coefficients are improved during unsharp masking algorithm at the back the Shearlet inverse transform process. [5], a novel image denoising technique based on Shearlet transform is future in this paper The original image is first decomposed use shearlet transform, after that the shrink threshold of every decomposed sub band adopt the different greatest threshold; produce the preface main denoised image after reconstruction. [6], this paper present a well-organized image denoising algorithm is include shearlet-based adaptive thresholding with non-local means filter. Firstly, adaptive Bayesian maximum a posteriori estimator where the normal reverse Gaussian distribution is use the earlier model of shearlet coefficients, is introduce for remove the Gaussian noise from corrupted image. Secondly, the nonlocal means filter is use to repress unnecessary no smooth artifact caused during the shearlet transform and shrinkage. [7], in this paper is the possibility of image fusion use shearlets. Fusion rules of bigger high-frequency coefficients founded on local energy, local variance, and absolute value are future as shearlet transform can catch exhaustive information in each scale and each direction. [8], this paper is a latest shearlet transform related through a no separable shearlet generator, which improves the directional selectivity of before shearlet transforms. advance is based on a discrete framework, which allow a realistic digitization of the scale domain directional transform based on efficiently support shearlets introduce as technique sparsely encode anisotropic singularities of multivariate data. Here explain numerical experiment representative the potential of our latest shearlet transform in 2D and 3D image processing application. [9], in this paper, is used a novel approach derived from the shearlet transform is multiscale directional transform with a better capability to localize distributed discontinuities for example edges. In fact, not like traditional wavelets, shearlets are in theory optimal in on behalf of images through edges and in particular contain the capability to completely imprison directional and other geometrical features. [10], in this paper a latest denoising algorithm obtained of translation-invariant non subsample Shearlet transform (NSST) use multistage products threshold is future. Following analyze the dependence of the NSST coefficients crossways scale space, and direction the adaptive multistage products of the NSST coefficients not directly apply to the NSST coefficients. [11], in this paper, a novel image denoising advance in extended Shearlet domain amid hidden Markov tree (HMT) model is future. Initially, the joint statistics and common information of the extend DST coefficients are study. [12], this paper present a novel image denoising technique by combining the shearlet shrinkage and enhanced total variation (TV). According to the artifact that disappears in the outcome image after apply shearlet denoising approach; the image is additional denoised by a TV model, which is enhanced on the reliability term.

\section{SHEARLET TRANSFORM}

Shearlet transform is a scale framework which allow to well encode anisotropic features within multivariate trouble module originally, shearlets was developed in 2006 for these are a innate sum of wavelet toward accommodate the reality to multivariate functions be typically govern with anisotropic features for example edges in images, on the other hand, wavelets similar to isotropic objects are unable of capture such phenomena. Shearlets is constructing by parabolic, scaling, shearing and translation to just a few generate function. Shearlets arise from the affine collection and allow a combined conduct of the continuum and digital condition leading to correct implementations. Although they do not constitute an orthonormal basis for the still form a frame allow stable expansion of arbitrary functions.

\subsection{Continuous shearlet systems:}

The construction of continuous shearlet systems is based on parabolic scaling matrix

$$
A_{a}=\left[\begin{array}{cc}
\mathbf{a} & \mathbf{0} \\
0 & \mathbf{a}^{1 / 2}
\end{array}\right], \mathbf{a}>\mathbf{0}
$$

as a mean to change the resolution, on shear matrices.

$$
S_{s}=\left[\begin{array}{ll}
1 & s \\
0 & 1
\end{array}\right], \quad s \in \mathbf{R}
$$

as a way to change the orientation, and finally on translations to change the position. In comparison to curvelets, shearlets use shearings instead of rotations, the benefit being that the shear operator $S_{S}$ leaves the integer lattice invariant in case $\mathrm{S}_{\mathrm{s}} \mathrm{Z}^{2} \subseteq \mathrm{Z}^{2}$. This really allows a unified treatment of the continuum and digital area there by guarantee a faithful digital implementation. For $\Psi \in \mathrm{L}^{2}\left(\mathrm{R}^{2}\right)$ the continuous shearlet system generate by $\Psi$ is then defined as $\mathrm{SH}_{\text {cont }}(\Psi)=$ $\left\{\Psi_{\mathrm{a}, \mathrm{s}, \mathrm{t}}=\mathrm{a}^{3 / 4} \Psi \quad\left(\mathrm{S}_{\mathrm{s}} \mathrm{A}_{\mathrm{a}}(-\mathrm{t})\right) \quad \mid \mathrm{a}>0, \quad \mathrm{~s} \in \mathrm{R}, \quad \mathrm{t} \in R^{2}\right\}$ and the corresponding continuous shearlet transform is specified by the map. $F \rightarrow \mathrm{SH}_{\Psi} f(\mathrm{a}, \mathrm{s}, \mathrm{t})=<f, \Psi_{\mathrm{a}, \mathrm{s}, \mathrm{t}}>, f \in \mathrm{L}^{2}\left(\mathrm{R}^{2}\right),(\mathrm{a}, \mathrm{s}, \mathrm{t})$ $\in \mathrm{R}_{>0} \mathrm{R} \times \mathrm{R}^{2}$.

\subsection{Discrete shearlet systems}

A discrete version of shearlet systems can be directly obtained from $\mathrm{SH}_{\text {cont }}(\Psi)$ by discretizing the parameter set $R_{>0} \mathrm{R} \times \mathrm{R}^{2}$. There are numerous approach for this but the most popular one is given by

$$
\left\{\left(2^{j}, \mathrm{k}, A_{2 J}^{-1}, S_{K}^{-1} \mathrm{~m}\right) \mid \mathrm{j} \in \mathrm{Z}, \mathrm{k} \in \mathrm{z}, \mathrm{m} \in \mathrm{z}^{2}\right\} \subseteq R_{>0} \mathrm{R} \times \mathrm{R}^{2} .
$$

From this, the discrete shearlet system associated with the shearlet generator $\Psi$ is defined by

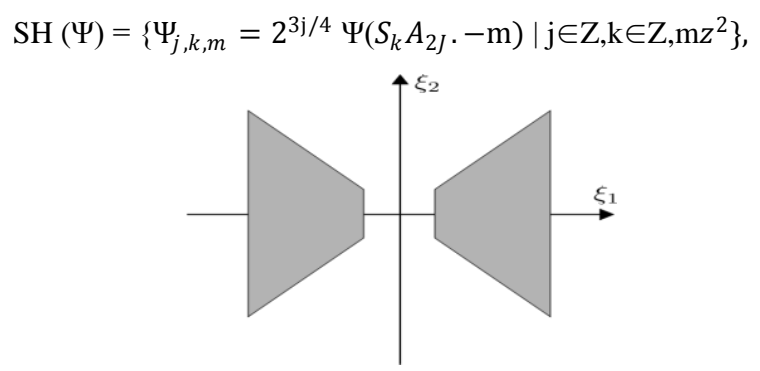

(a)

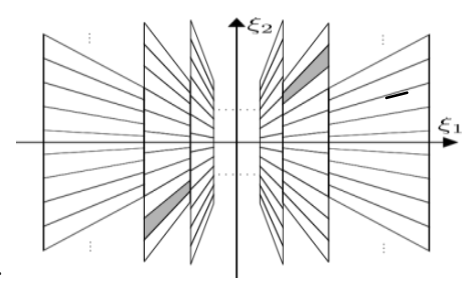

(b)

Fig.1 (a) Trapezoidal frequency support of the classical shearlet and (b) Frequency tiling of the (discrete) classical shearlet system. 


\section{BAYESIAN NETWORK}

Bayesian networks (BNs) or Bayes nets for small, also called belief networks, belong to the group of probabilistic graphical model (GMs). These graphical structures are utilized to represent information, knowledge as well as relations about an uncertain domain. In the BNs graph, each node represent a random variable, where since the edges between the nodes represent probabilistic dependencies/relations in the middle of the corresponding/linked random variable. These conditional dependencies into the graph are estimated by different statistical and computational methods. And so BNs structure can be defined like a combine theory of graph, probability, computer science, and statistics [13].

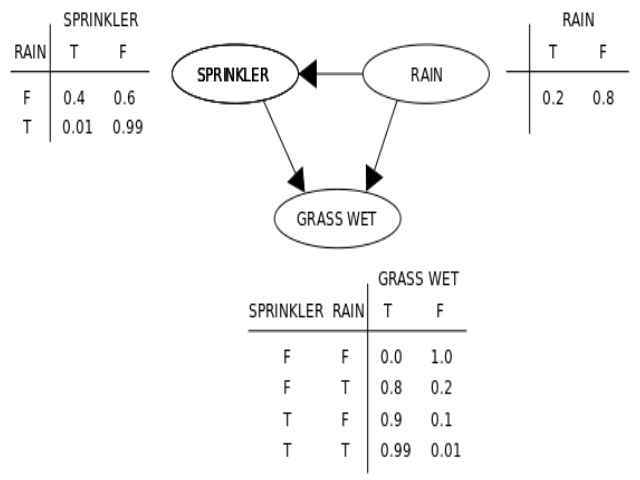

Fig. 2 structure of the Bayesian Network

Follow the above discussion, an additional formal definition of a BN can be derived as: Let a Bayesian network B is an annotated acyclic graph that represent JPD(joint probability distribution) for a set of random variablesV. The network is defined by a couple

$$
\mathrm{B}=\langle\mathrm{G}, \theta\rangle
$$

Where G is the DAG (directed acyclic graph) whose nodes?

$$
\mathrm{X}_{1}, \mathrm{X}_{2}, \ldots, \mathrm{X}_{\mathrm{n}}
$$

Represent random variables, plus whose edges represent the direct dependencies linking these variables. The graph $G$ encodes independence assumptions; by which each variable $\mathrm{X}_{\mathrm{i}}$ is independence of it's no descendent given its parents in $\mathrm{G}$. The next component $\theta$ denotes the set of parameter

$$
\theta_{\mathrm{x}_{\mathrm{i}} \mid \pi_{\mathrm{i}}}=\mathrm{P}_{\mathrm{B}}\left(\mathrm{x}_{\mathrm{i}} \mid \pi_{\mathrm{i}}\right)
$$

For each realization $x_{i}$ of $\mathrm{X}_{\mathrm{i}}$ conditioned on $\pi_{\mathrm{i}}$, set of parents' of $\mathrm{X}_{i}$ in G.accordingly, B defines a unique JPD over $\mathrm{V}$, namely:

$$
\mathrm{P}_{\mathrm{B}}\left(\mathrm{X}_{1}, \mathrm{X}_{2}, \ldots, \mathrm{X}_{\mathrm{n}}\right)=\prod_{\mathrm{i}=1}^{\mathrm{n}} \mathrm{P}_{\mathrm{B}}\left(\mathrm{x}_{\mathrm{i}} \mid \pi_{\mathrm{i}}\right)=\theta_{\mathrm{x}_{\mathrm{i}} \mid \pi_{\mathrm{i}}}
$$

For ease of representation we leave out the subscript B in future. If $\mathrm{X}_{\mathrm{i}}$ has no parents, its local probability distribution is said to be unconditional, otherwise it is conditional. If the variable represented by a node is observed, next the node is said to be an evidence node, other wise the node is said to be hidden and latent.

\section{PROPOSED ALGORITHM}

The proposed algorithm is based on the Shearlet transform and Bayesian network. The complete algorithm can be explained as follows.
Step1: firstly the noisy image is transformed into shearlet domain.

Let $\mathrm{I}_{\text {noisy }}$ be the noisy image then

$\mathrm{S}=\left\{\mathrm{S}_{\mathrm{avg}}, \mathrm{S}_{\mathrm{A}}, \mathrm{S}_{\mathrm{B}}, \mathrm{S}_{\mathrm{C}}, \mathrm{S}_{\mathrm{D}}\right\}=$ funSharelet $\left(\mathrm{I}_{\text {noisy }}\right)$;

Where

$\mathrm{S}_{\text {avg }}$

= Low - pass coefficent to first decomposition index.

$\mathrm{S}_{\mathrm{A}}, \mathrm{S}_{\mathrm{B}}, \mathrm{S}_{\mathrm{C}}, \mathrm{S}_{\mathrm{D}}$ are the directional components.

$\mathrm{S}_{\text {avg }}, \mathrm{S}_{\mathrm{A}}, \mathrm{S}_{\mathrm{B}}, \mathrm{S}_{\mathrm{C}}, \mathrm{S}_{\mathrm{D}}$ and $\mathrm{I}_{\text {noisy }}$ have the same size of $\mathrm{N}^{*} \mathrm{~N}$

Step2: Create a vertex set $\left(\mathrm{V}^{\mathrm{u}}\right)$ of size $\mathrm{m}^{*} \mathrm{~m}$ by sampling the each component of $\mathrm{S}=\left\{\mathrm{S}_{\mathrm{avg}}, \mathrm{S}_{\mathrm{A}}, \mathrm{S}_{\mathrm{B}}, \mathrm{S}_{\mathrm{C}}, \mathrm{S}_{\mathrm{D}}\right\}$, at the interval $\mathrm{N} / \mathrm{m}$.

Step3: Create a sibling edges $\left(\mathrm{E}_{\mathrm{i}}^{\mathrm{u}}\right)$ for nodes at the same scale and orientation by

1. Calculating the empirical probability $\left(\mathrm{P}_{\mathrm{i}}^{\mathrm{u}}\right)$ of each variable from the frequency counts of square of $(\mathrm{N} / \mathrm{m})$ shearlet coefficient assigned to the node.

2. Generation of maximal weighted spanning tree from the $\mathrm{m}^{2}$ node in each sub-band

Step4: Create inter-scale edge $\left(E_{0}^{\mathrm{u}}\right)$ at each orientation in same way as $\left(E_{i}^{u}\right)$.

Step5: Calculate V, E and P

$\mathrm{V}=\mathrm{V}_{\text {avg }} \cup \mathrm{V}_{\mathrm{A}} \cup \mathrm{V}_{\mathrm{B}} \cup \mathrm{V}_{\mathrm{C}} \cup \mathrm{V}_{\mathrm{D}}$;

E $\quad E_{\text {avg }} \cup E_{A} \cup E_{B} \cup E_{C} \cup E_{D}=$;

$\mathrm{P}=\mathrm{P}_{\mathrm{avg}} \cup \mathrm{P}_{\mathrm{A}} \cup \mathrm{P}_{\mathrm{B}} \cup \mathrm{P}_{\mathrm{C}} \cup \mathrm{P}_{\mathrm{D}}$

Step6: Create the Shearlet Bayesian Network from each of $\mathrm{V}, \mathrm{E}, \mathrm{P}$ calculate

$$
\mathrm{SBN}=\text { funBN }(\mathrm{V}, \mathrm{E}, \mathrm{P}) \text {; }
$$

Step7: Estimate the original coefficient from noisy coefficient in S

$\left\{S_{\text {avg }}^{\text {denoised }}, S_{A}^{\text {denoised }}, S_{B}^{\text {denoised }}, S_{C}^{\text {denoised }}, S_{D}^{\text {deno ised }}\right\}=$ fun

Est (SBN, $\left.\mathrm{S}_{\mathrm{avg}}, \mathrm{S}_{\mathrm{A}}, \mathrm{S}_{\mathrm{B}} \mathrm{S}_{\mathrm{C}}, \mathrm{S}_{\mathrm{D}}\right)$

Step 8: take the inverse Shearlet Transform to get the denoised image $\mathrm{I}_{\text {denoised }}=$

Fun InvSharelet $\left(\mathrm{S}_{\text {avg }}^{\text {denoised }}, \mathrm{S}_{\mathrm{A}}^{\text {denoised }}, \mathrm{S}_{\mathrm{B}}^{\text {denoised }}, \mathrm{S}_{\mathrm{C}}^{\text {denoised }}\right.$, $\mathrm{S}_{\mathrm{D}}^{\text {denoised }}$ ) 


\section{Flow Chart of Proposed algorithm}

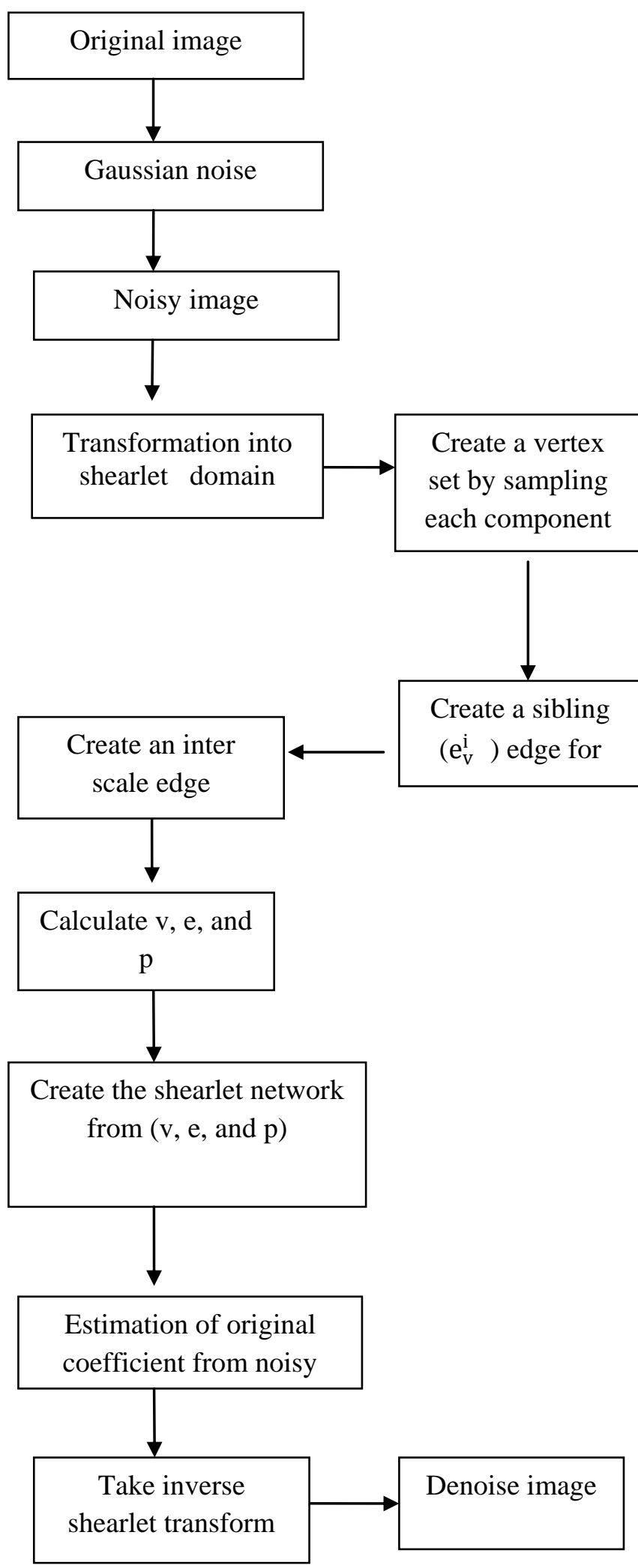

\section{SIMULATION RESULT}

This present algorithm is tested and compare for dissimilar gray scale images (Shown in Figure 4) of size 256x256 pixels corrupted by means of different noise density (from 0.01 to 0.05 ) with wavelet based technique used for the performance analysis.
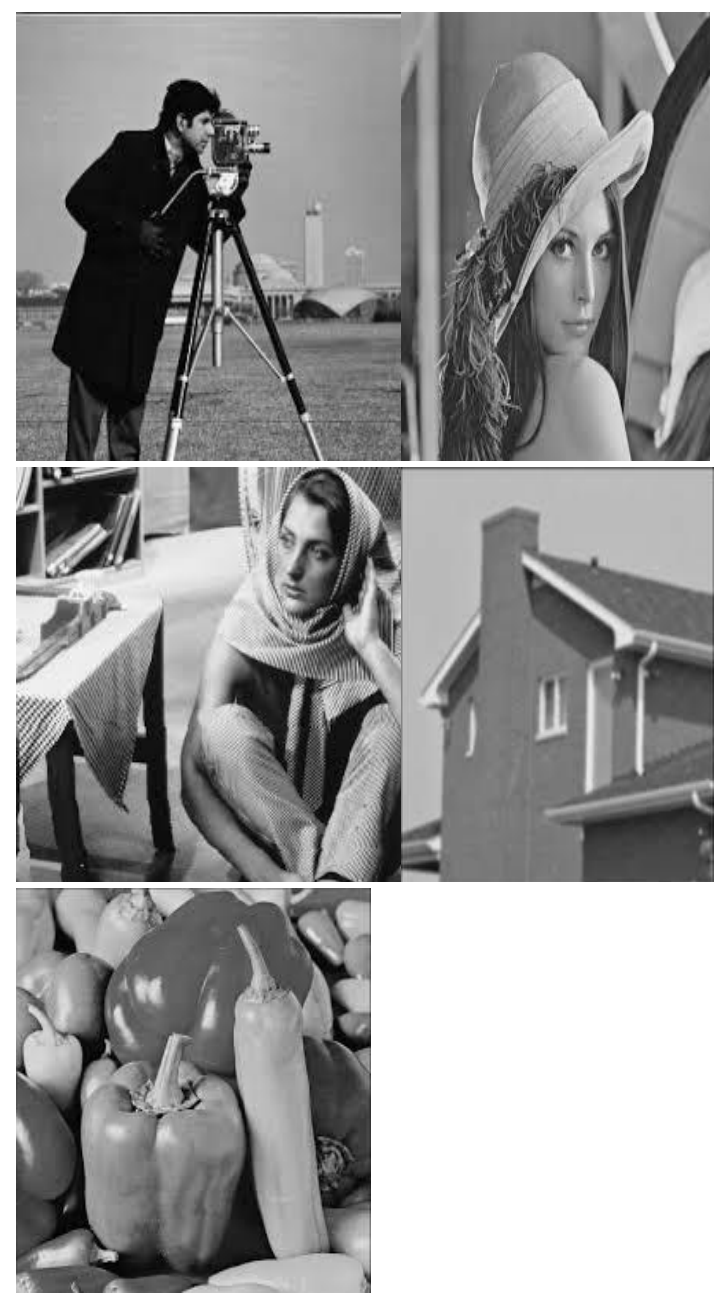

Fig. 3 Test Images used in the Analysis, Cameraman, Lena, Barbara, House, pepper

\section{FLOW CHART OF PROPOSED ALGORITHM}


Table: Test images used in the analysis, Cameraman, Lena, Barbara, house, pepper

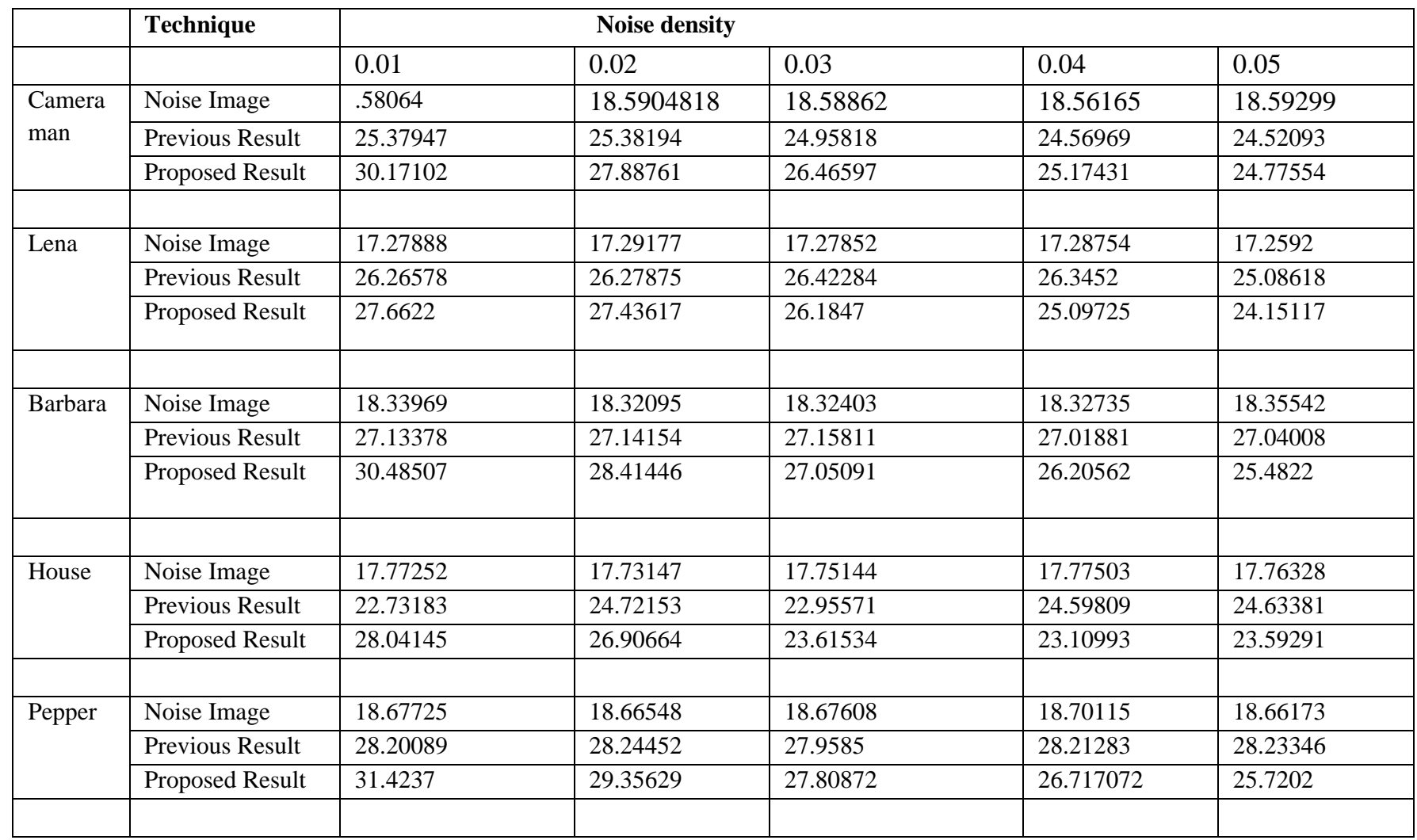

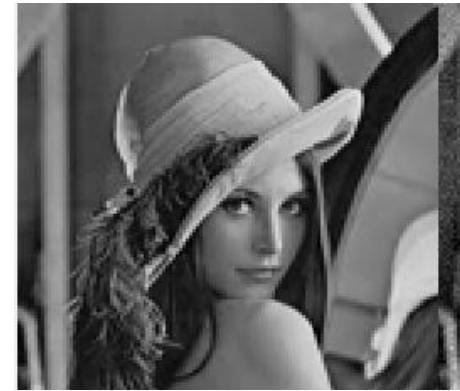

Fig.4 Original Image

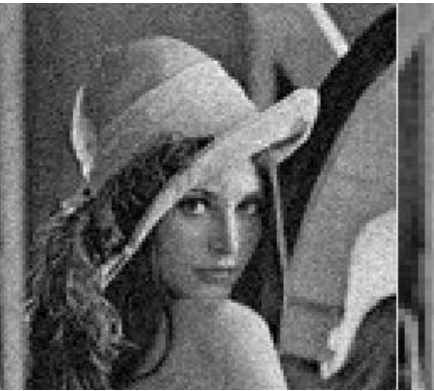

Image Corrupted By $\sigma^{2}=20$ Filtered

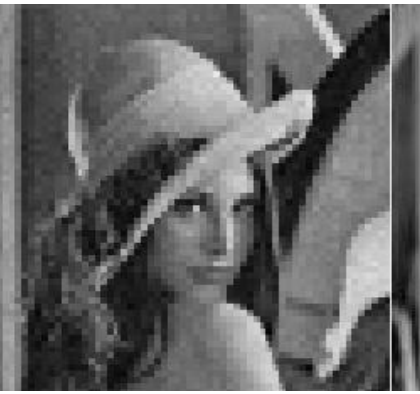

Image by Wavelet

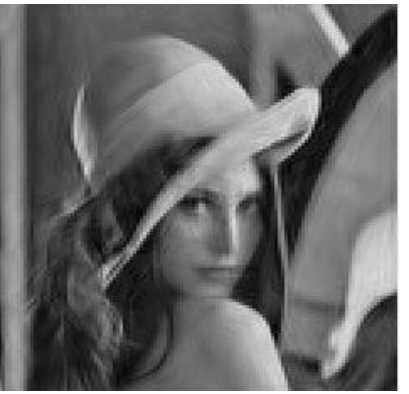

Filtered Image By Proposed

\section{CONCLUSION}

The simulation results of the proposed Shearlet Transform and Bayesian Network based denoising technique show that for the image denoising problem the noisy coefficients estimation be able to be model because probability model as well as estimation task. This paper also shows that adaptability of the wavelet based thresholding technique with shearlet. Presented procedure and provide the training based filtering which is especially useful for the cases where the mathematical model of noise is impossible and can also be trained for dissimilar types of noises. Finally the comparison of proposed denoised results with individuals derived by wavelet approach demonstrate the future technique gives improved PSNR and improved perceptual quality on the textured and non-textured areas of an image ways to speed up our algorithm's execution time is an problem that advantage future study.

\section{REFERENCES}

[1] Chengzhi Deng, Saifeng wei Tian "Total Variation Based Multivariate Shearlet Shrinkage For Image Reconstruction", Institute of Advanced Engineering and science Telkomnika, Vol. 11, January2013, pp. 40-47.

[2] Miao, Qi-guang, Shi, Cheng "novel algorithm of image fusion using shearlets",2011 Optics Communications, Volume 284, Issue 6, p. 1540-1547.

[3] Glenn Easley ${ }^{\mathrm{a}}$, Demetrio Labate ${ }^{\mathrm{b}}$,Wang-Q Lim ${ }^{\mathrm{c}}$."Sparse directional image representations using the discrete shearlet transform" Volume 25, Issue 1, July 2008 Pages 25-46.

[4] Wubuli, Ayiguli, Zhen-Hong, Jia; Xi-Zhong, Qin; Jie, "Medical Image Enhancement Based on Shearlet 
Transform and Unsharp Masking”, Journal of Medical Imaging and Health Informatics, Volume 4, Number 52014.

[5] J. Zhao, L. Lü, H. Sun, "Multi-Threshold Image Denoising Based on Shearlet Transform", Applied Mechanics and Materials, Vols. 29-32, pp. 2251-2255, Aug. 2010.

[6] Chengzhi Deng, Wei Tian, Saifeng Hu, Yan Li, Min Hu, Shengqian Wang, "Shearlet-based image denoising using adaptive thresholding and non-local means filter", International Journal of Digital Content Technology and its Applications(JDCTA) Volume6,Number20,November 2012.

[7] Qi-guang Miao, Cheng Shi, "A novel algorithm of image fusion using shearlets",Volume 284, Issue 6, 15 March 2011, Pages 1540-1547.

[8] Wang-Q Lim, "Non separable Shearlet Transform", Image Processing, IEEE Transaction 2013 (Volume: 22 Issue: 5 )

[9] Sheng Yi, Labate, D. ; Easley, G.R. ; Krim, H. "A
Shearlet Approach to Edge Analysis and Detection", Image Processing, IEEE Transactions on (Volume:18, Issue:5) March 2009.

[10] Junliang Liu; Lin Lei; Shilin Zhou. "Nonsubsampled Shearlet-based image denoising using multiscale products",

[11] Intelligent Control and Information Processing (ICICIP), 2013 Fourth International Conference on. YangChengLiua,Hong-YingYanga, "Image denoising in extended Shearlet domain using hidden Markov tree models" Volume 30, July 2014, Pages 101-113.

[12] Ying Li, Rui-ming, Chen Shi Liang. "A New Image Denoising Method Based on Shearlet Shrinkage and Improved Total Variation".Volume7202 of the series Lecture Notes in Computer Science pp 382-388

[13] Ben-Gal I., Ruggeri F., Faltin F. \&Kennett R. "Bayesian Networks", Encyclopedia of Statistics in Quality \& Reliability, Wiley \& Sons (2007). 